\title{
RELACIONES ENTRE \\ CONCEPTUALIZACIÓN Y USO EN EL APRENDIZAJE DE LA GRAMÁTICA. DOS INVESTIGACIONES DE AULA SOBRE EL VERBO
}

RESUMEN: La enseñanza de la gramática necesita considerar el aprendizaje de los alumnos, sus logros y dificultades en la apropiación de los conceptos gramaticales, para poder intervenir con eficacia en las aulas. Es decir, necesita entender la actividad metalingüística que desarrollan los estudiantes, tanto en actividades de reflexión gramatical en sí mismas como en otras en que la gramática entra en relación con la escritura. Desde esta perspectiva, el artículo presenta dos investigaciones sobre la enseñanza del verbo en Educación Secundaria Obligatoria (12-16 años): sobre el uso de los tiempos verbales del pasado en la escritura y en torno a la conceptualización del subjuntivo. En ambas se analiza cómo utilizan los alumnos sus conocimientos lingüísticos bien en la reflexión bien en la escritura. Estos datos permiten avanzar en una gramática pedagógica que tenga en cuenta los problemas de la enseñanza y el aprendizaje al realizar la transposición didáctica de los contenidos gramaticales.

PALABRAS CLAVE: actividad metalingüística, reflexión gramatical, conceptos gramaticales, enseñanza del verbo

ABSTRACT: Grammar teaching needs to take into students' learning, their achievements and difficulties in the understanding of grammatical concepts to be able to intervene effectively in classroom. That is, it is necessary to understand what kind of metalinguistic activity students develop, both in grammatical reflection activities and in others in which grammar is related to writing. From this perspective, the article presents two studies about how students of Compulsory Secondary Education (12-16 years) conceptualize 
the verb. In the first study we explore their conceptualization in relation to the use of past tenses in writing. In the second study we explore their views concerning the notion of subjunctive. In both we analyse how students use their linguistic knowledge in reflection and in writing. We expect that these results enable us to advance in a pedagogical grammar, which takes into account the problems of teaching and learning when making the didactic transposition of grammatical contents.

KEYWORDS: metalinguistic activity, grammatical reflection, grammatical concepts, verb teaching

\section{1 - La enseñanza de la gramática}

La enseñanza y el aprendizaje de la gramática en la educación obligatoria se ha planteado en muchas ocasiones como un proceso unidireccional de adaptación o de simplificación de las aportaciones de las teorías lingüísticas a los niveles educativos básicos. Ya en 1985 Bronckart advertía del peligro de que la enseñanza "corriera" en pos de los últimos avances de las ciencias del lenguaje y señalaba que habían de ser los fines de la enseñanza los que determinaran la relación con las ciencias de referencia. También en esa época, y desde la didáctica de las matemáticas, Chevallard (1985) acuñaba el concepto de transposición didáctica para entender la relación entre las ciencias de referencia y el contenido didáctico.

Mucho se ha avanzado en las didácticas específicas desde entonces. En el caso que nos ocupa, la didáctica de la gramática, también se ha recorrido un largo camino no exento de polémicas (Locke 2010; Fontich \& Camps 2014), centrado en la necesidad de conocimientos gramaticales explícitos y en su incidencia en el dominio de la lengua, especialmente en los usos escritos. En torno a estas cuestiones, Camps (2000) plantea el concepto de actividad metalingüística, referido a toda aquella actividad que el hablante realiza, con distintos grados de conciencia y explicitud, para resolver problemas de uso del lenguaje, y para explicitar sus conocimientos y sus observaciones sobre el mismo, tanto en lenguaje común como mediante metalenguaje. El concepto de actividad metalingüística permite superar dos planteamientos contrapuestos: la enseñanza de la gramática explícita - con tradición en España, Francia, Italia, Portugal, entre otros- frente a la enseñanza implícita, que se desprende directamente del uso -en países anglosajones, principalmente.

Desde nuestra perspectiva, se trata de considerar la enseñanza de la gramática como reflexión sobre la lengua, es decir como la parte del desarrollo de la facultad metalingüística de los hablantes que se realiza en el ámbito escolar y que considera tanto la reflexión sobre el uso de la lengua como la 
necesidad de conceptos que permitan esta reflexión. No se entiende, pues, ni como una pura manipulación inducida de estructuras gramaticales ni como una memorización de definiciones aplicadas a ejemplos estereotipados. Enseñar a los alumnos a reflexionar sobre la lengua, es decir, a convertir el lenguaje en opaco, más allá de las diversas finalidades comunicativas con que lo utilizamos a diario, permite a los estudiantes un control progresivo, especialmente en relación con los usos formales.

En este sentido, es necesario un planteamiento metodológico que tenga en cuenta los obstáculos con que se encuentran los alumnos (Fisher 2004; Camps 2009) y desarrolle una tipología de actividades gramaticales que propicien la reflexión metalingüística en el aula. Entre estas actividades, a modo de ejemplo, citamos la reflexión a partir de pares mínimos (Bosque 1994), la construcción de procedimientos para aplicar normas o para detectar errores (Chartrand 2003) o la manipulación de enunciados mediante conmutación, supresión, ampliación con explicación de los efectos que producen (Zayas 2011). Asimismo, y atendiendo también al cambio metodológico, Camps (2003) propone las secuencias didácticas para aprender gramática (en adelante, SDG), que permiten articular estas actividades en intervenciones de aula sobre contenidos gramaticales de diverso tipo (Camps \& Zayas 2006) entendidas como procesos de indagación y reflexión sobre la lengua en los que la interacción de los estudiantes (entre sí y con el profesor) desempeña un papel fundamental en el aprendizaje.

Es decir, hablar de enseñanza de la gramática implica tener en cuenta los tres polos del sistema didáctico: el alumno y sus modos de aprendizaje; el profesor y los modos de enseñanza; y los contenidos gramaticales y sus distintos planteamientos desde las ciencias de referencia. De ahí que una gramática pedagógica haya de ser diferente de las gramáticas divulgativas - como la gramática básica de la RAE o la gramática descomplicada de Grijelmo (2006), ambas para el español, por poner un par de ejemplos-, que se dirigen a usuarios ya formados (en la formación básica, como periodistas...) y plantean unas nociones ya conocidas por ellos. Así se indica en el prólogo de la Nueva gramática básica de la lengua española (2011: XVII), "[Esta gramática] se dirige al amplio espectro de hispanohablantes que, habiendo recibido la primera instrucción en sus estudios de primaria y secundaria, deseen acercarse a comprender mejor el funcionamiento de su lengua". La gramática pedagógica también se diferencia de las gramáticas dirigidas a estudiantes de lenguas extranjeras, que necesitan contrastar las nociones básicas con las ya construidas en su primera lengua, bien de forma espontánea a partir del uso, bien mediante enseñanza gramatical explícita.

Al referirnos a la enseñanza de lenguas primeras, la gramática pedagógica tiene como finalidad básica guiar al estudiante en la construcción de este 
primer conocimiento sistemático de la lengua, que le permita una progresiva regulación de los usos formales, fundamentalmente en la escritura. Es decir, se trata de "dotar al alumnado de conceptos y estrategias para indagar reflexivamente sobre la lengua, tanto desde la vertiente del sistema gramatical como desde la vertiente del uso" (Fontich 2017: 9). Esta es la línea en la que se sitúa el trabajo del Grup de Recerca sobre l'Ensenyament i l'Aprenentatge de la Llengua (Greal) ${ }^{1}$, dos de cuyas investigaciones sirven de base a este artículo. En ellas, el análisis de la actividad metalingüística de los estudiantes en tareas de reflexión gramatical muestra la necesidad de plantear un trabajo gramatical en el aula que guíe al estudiante en el proceso de abstracción que supone la adquisición de las categorías gramaticales.

\section{2 - Las categorías gramaticales como conceptos}

\section{1 - Las categorías gramaticales y el proceso de abstracción}

Las categorías gramaticales, como otros conceptos científicos que los estudiantes tienen que aprender en la escuela, son nociones muy abstractas, que requieren de un proceso de construcción largo y laborioso no exento de dificultades (Fisher 2004; Camps 2009). Pensemos, por ejemplo, en la noción de morfema, una noción básica cuando se aborda la enseñanza de la estructura interna de las palabras. El morfema es un signo lingüístico $\mathrm{y}$, como tal, implica una forma y un significado. Ahora bien, los morfemas no tienen la independencia de una palabra e incluso pueden no tener una materialización concreta como es el caso de los morfemas verbales de tiempo, aspecto y modo, que aparecen fusionados en las lenguas románicas (Pérez Saldanya 2017). Esta característica hace difícil el reconocimiento de estas nociones, primer estadio en un proceso de abstracción (Barth 2001; van Hiele 1986) que permita su conceptualización.

Asimismo, de nuevo siguiendo a Barth (2001), es necesario distinguir entre la formación de conceptos y la adquisición de conceptos. En el primer caso, se trata de un proceso creativo espontáneo en que los individuos reagrupan los elementos según sus propios criterios y su conocimiento del mundo. En el segundo, en cambio, la regla de clasificación viene dada de manera externa y los conceptos forman parte de una teoría de referencia, de una construcción teórica ya existente. Esta distinción procede de la diferencia entre conceptos espontáneos y conceptos científicos establecida por Vigotski (1995), según la cual estos últimos han de formar parte de sistemas para poder ser recuperables de forma consciente y, para ello, han de

\footnotetext{
${ }^{1}$ Grupo de investigación fundado por Anna Camps, con sede en la Universitat Autònoma de Barcelona, y que agrupa investigadores también de la Universitat de València y de la Universitat de Vic-Universitat Central de Catalunya.
} 
presentarse como objetos de aprendizaje consciente (Camps 2017). Es decir, no es suficiente con que los estudiantes observen cómo funciona la lengua en uso, sino que se requiere de una mediación didáctica que les acompañe en el proceso de adquisición del concepto en el marco de un sistema de relaciones con otros conceptos.

Además, las categorías gramaticales se pueden interpretar como compartimentos estancos $-\mathrm{y}$ por lo tanto fáciles de delimitar las unas de las otras- o, siguiendo los postulados de la lingüística cognitiva (Lakoff 1987; Langacker 1987; Cuenca \& Hilferty 1999), se puede considerar que los límites entre las categorías son difusos y permeables. Por ello, se distingue entre miembros prototípicos y miembros periféricos de una misma categoría. El prototipo sería "el ejemplar que mejor se reconoce, el más representativo y distintivo de una categoría, puesto que es el que comparte más características con el resto de los miembros de la categoría y menos con los miembros de otras categorías" (Cuenca \& Hilferty 1999: 35). Por ejemplo, el pino sería un miembro prototípico de árbol puesto que reúne todas las características que se asocian a ellos. Los ejemplos periféricos, en cambio, presentan fronteras difusas con otras categorías. Así, un bonsái se podría considerar un miembro periférico, puesto que su pequeño tamaño lo hace más cercano a una planta que a un árbol.

Aplicada a las categorías gramaticales, esa distinción nos puede ayudar a entender las dificultades que tienen los estudiantes para identificar una categoría concreta o bien para comprender la noción, sobre todo si-como muestran diversos estudios (Durán 2010; 2012a en relación con el adverbio y el verbo, respectivamente; Notario 2001, en relación con el sujeto)- en su enseñanza solo se tienen en cuenta los ejemplos prototípicos y no los ejemplos periféricos de una misma categoría, lo que dificulta que se comprenda la complejidad de la noción. Por ejemplo, en el trabajo de Durán (2012a), los estudiantes -alumnos de $4^{\circ}$ de la Educación Secundaria Obligatoria, 15-16 años- definen la categoría verbo como una palabra que indica acción; es decir, remiten en su definición a un ejemplo prototípico, lo que obstaculiza la comprensión de la noción en su totalidad puesto que deja fuera muchos otros ejemplos.

En este proceso de conceptualización, la interacción es clave, como señala Lemke (1997), quien plantea el aprendizaje de la ciencia -y de los conceptos científicos- a través de la interacción social y del lenguaje como instrumento al servicio del aprendizaje. Para este autor, "hablar ciencia" (talking science) es apropiarse de los conocimientos científicos a través de la mediación del lenguaje (learning through language). No se trata solo de la utilización de unos términos u otros; la construcción de conceptos tiene que ir acompañada de la construcción de sistemas conceptuales sin los cuales los 
primeros no se pueden entender. En el contexto del aprendizaje gramatical, Milian (2005), siguiendo a Lemke, hace referencia a la función cognitiva y constructiva de la lengua en situaciones de negociación colectiva y a la importancia de hablar para "hacer gramática" con el objetivo de conseguir que los aprendices se apropien del conocimiento gramatical.

\section{2 - Una noción clave: el verbo}

El verbo es una de las categorías clave que una gramática escolar tiene que abordar, tanto en lo que se refiere al conocimiento de la noción y su relación con otras categorías, como por la función fundamental que tiene en la escritura (Casas, Durán \& Fontich 2017). Algunas investigaciones se han centrado en las dificultades que puede suscitar este contenido gramatical en la enseñanza de lenguas. Estos trabajos muestran que el concepto de verbo se encuentra en proceso de adquisición a lo largo de toda la escolaridad (Garitte 2004); que los estudiantes lo reconocen a menudo en función del contexto en el que se encuentra y que solo a partir de los últimos años de la Primaria (11-12 años) la mitad de los estudiantes identifican las formas verbales descontextualizadas (Tisset 2004). También constatan que la construcción de la noción es compleja, que implica ritmos individuales diferenciados (Roubaud \& Touchard 2004) y que a menudo los estudiantes se limitan a memorizar el paradigma sin haberlo comprendido (Lusetti 2008).

Estas dificultades detectadas en la educación primaria, no parece que se solucionen en la educación secundaria. El trabajo de Durán (2012a) sobre la representación de la categoría verbo de estudiantes de la Secundaria Obligatoria muestra que al final de la etapa (16 años) los estudiantes identifican con gran seguridad el verbo en sus formas simples, pero no en las formas compuestas o en las formas no personales. Muchos de ellos, sin embargo, no son capaces de reconocer los tiempos verbales y se sitúan en un eje cronológico básico (pasado, presente y futuro) en el que confunden el tiempo gramatical y el tiempo extralingüístico. En cuanto a los saberes declarativos, los estudiantes definen la categoría por acumulación de rasgos, sobre todo formales y semánticos, pero no parece que hayan sistematizado este conocimiento. Han creado una noción fosilizada del verbo como palabra que indica acción, que les obstaculiza la reflexión cuando se encuentran ante verbos que no tienen ese significado.

En esta línea, diversos estudios coinciden en la necesidad de dotar de instrumentos a los estudiantes para crear la noción desde una aproximación reflexiva, que atienda a la complejidad del verbo como objeto de enseñanza y que tenga en cuenta los conocimientos previos de los estudiantes (Fontich 2006; Martínez 2010; Torralba 2012; Casas 2014). 
$\mathrm{Y}$ es que el verbo es la categoría léxica flexiva más compleja en las lenguas románicas (Pérez Saldanya 2017: 33). Frente a la variación de género y número del sustantivo, el verbo dispone de variaciones de persona, número, tiempo, aspecto y modo -si contamos todas las formas flexivas diferentes que puede adoptar un solo verbo en español, entendido este como palabra léxica o entrada de diccionario, nos encontramos con 65 formas simples y 121 formas compuestas- $\mathrm{y}$ tiene un contenido semántico diverso (acciones, eventos y estados). La complejidad de la flexión verbal se debe a que aporta información de diferentes propiedades gramaticales al mismo tiempo (persona, número, tiempo, modo, aspecto), pero también a que el usuario tiene que distinguir entre el tiempo lingüístico y el tiempo extralingüístico y utilizar las distintas formas según el momento de la enunciación (pasado o presente). Todo ello dificulta la comprensión de la noción y hace necesaria una enseñanza que introduzca progresivamente su complejidad.

\section{3 - Dos aproximaciones a la noción de verbo}

\section{1 - El problema de investigación: los tiempos del pasado y el modo verbal}

Con estas premisas, nos proponemos mostrar algunos aspectos de dos investigaciones de aula en torno a la categoría gramatical del verbo, realizadas ambas con alumnos de Educación Secundaria Obligatoria (12-16 años)².

La primera de estas investigaciones (Rodríguez Gonzalo 2014a) parte del diseño de una secuencia didáctica de gramática (SDG) como espacio didáctico de reflexión sobre la lengua, organizado a partir de la pregunta ¿para qué sirven los tiempos verbales del pasado? Para responder a la pregunta, se plantea un proceso de indagación y reflexión con dos tareas centrales: una primera, de conceptualización sobre los tiempos verbales del pasado seguida de una tarea de escritura, el relato de una experiencia vivida. En el primer caso, se plantea el conocimiento de los distintos tiempos verbales desde la reflexión sobre sus semejanzas y sus diferencias, según los planteamientos de Barth (2001) en relación con la enseñanza de la abstracción. De esta forma, se pretende que puedan explicar, por ejemplo, las diferencias entre tiempos como pasó (pretérito perfecto simple) y había pasado (pretérito pluscuamperfecto) en contextos de alternancia como Perdió el libro que se compró pocos días antes / Perdió el libro que se había comprado pocos días antes.

En la tarea de escritura se persigue que los estudiantes utilicen conscientemente el complejo paradigma verbal del español. Para ello,

\footnotetext{
${ }^{2}$ En España, la Educación Secundaria Obligatoria (ESO) comprende cuatro cursos. El primero corresponde a alumnos de 12-13 años y el cuarto, de 15-16 años.
} 
se guía tanto la planificación como la revisión entre iguales anterior a la versión final del texto. En ambos casos, la interacción en aula, entre iguales (actividad de conceptualización en pareja o de planificación en pequeño grupo) y con el profesor (revisión) permite a los estudiantes hacer emerger la actividad metalingüística que realizan. En la investigación, el contraste entre los textos (borradores y versiones finales) y las interacciones de aula (triangulación, en términos de investigación cualitativa) permite entender cómo se relaciona la conceptualización y el uso escrito en la enseñanza de la gramática.

En el segundo caso (Durán 2015a), la investigación se centra en una tarea de reflexión metalingüística sobre los valores del subjuntivo a partir de cuatro enunciados de pares mínimos (Bosque 1994) cuya única diferencia estriba en la selección modal entre indicativo y subjuntivo, como en (a) Busco a una persona que sabe italiano / (b) Busco a una persona que sepa italiano. Partiendo del uso, se investiga sobre cómo estudiantes de la Secundaria Obligatoria han construido el concepto de modo subjuntivo en la educación obligatoria, una noción clave para entender el paradigma verbal en español y los valores modalizadores de la selección modal, así como para observar el papel de la actividad metalingüística en el aprendizaje de los conceptos gramaticales.

En este caso, el foco de la investigación se pone en el propio sistema lingüístico a partir de una tarea de análisis guiada a través de una pauta. Los estudiantes tienen que elaborar en grupo un pequeño informe tras discutir entre ellos la respuesta a estas preguntas: ¿cuál es la diferencia entre el par de oraciones a y b?, ¿significan lo mismo?, ¿qué palabra cambia?, ¿en qué consiste el cambio?, ¿por qué seleccionamos una forma $\mathrm{u}$ otra? Las preguntas focalizan tanto en el significado como en la forma. El diseño del instrumento de recogida de datos responde al objetivo de investigación de hacer emerger los conocimientos declarativos sobre el modo verbal y de elicitar la actividad metalingüística sobre este contenido gramatical para observar cómo los estudiantes interpretan la diferencia entre las formas verbales de indicativo y de subjuntivo, qué noción de modo verbal emerge de sus respuestas y con qué dificultades se encuentran.

Como hemos señalado al principio, las dos investigaciones se inscriben en la línea de trabajo del grupo Greal en relación con la actividad metalingüística y el aprendizaje de la gramática, y en ambos casos, la interacción verbal -hablar para aprender gramática- ha sido un instrumento esencial de la investigación, ya que ha permitido entender el nivel de conceptualización real de los alumnos, esencial para reorientar la intervención didáctica. El objetivo último de estas investigaciones es 
crear conocimiento sistemático sobre cómo construyen los alumnos de Secundaria el saber gramatical para mejorar los procesos de enseñanza y aprendizaje de la gramática.

\section{2 - El uso de los tiempos verbales del pasado}

La primera investigación está guiada por dos preguntas básicas: ¿cómo conceptualizan los estudiantes las nociones gramaticales, en este caso, las diferencias entre los tiempos verbales del pasado?, ¿el saber gramatical les sirve a los estudiantes para escribir mejor?

Como es sabido, en español, las variaciones temporales y aspectuales en las formas verbales (tiempos verbales) configuran un complejo sistema que, en el uso, se articula en torno de los ejes básicos de enunciación, el presente y el pasado. Esta articulación es la llamada consecutio temporum (Weinrich 1974; Carrasco 1999) y resulta posible porque el tiempo es, a la vez, una propiedad deíctica -por referencia directa o indirecta al acto de enunciación- y de orientación, ya que permite establecer relaciones de anterioridad, posterioridad y simultaneidad, respecto del momento de enunciación elegido. Por su parte, el aspecto, al hacer referencia al tiempo interno de la acción o al estado expresado por el verbo, indica si se trata de una situación en curso de realización, de una situación ya culminada o que se repite con regularidad, etc. Los tiempos verbales pueden expresar algunas de estas nociones aspectuales y en este sentido se habla de tiempos imperfectivos (como el pretérito imperfecto) y perfectivos (el pretérito perfecto simple y todos los compuestos con haber) (Pérez Saldanya 2007: 43-44).

Dominar este complejo sistema no es fácil, de ahí que, como señala López García (2005: 162), la mayoría de los hispanohablantes utilice básicamente las cinco formas verbales simples (canto, canté, cantaba, cantaré, cantaría). Esta situación cambia al escribir, porque es necesario precisar las relaciones de anterioridad, simultaneidad y posterioridad que se dan respecto de los momentos básicos de presente y pasado.

Nuestra investigación surge de la observación de los problemas de los alumnos de Secundaria para utilizar formas de uso elaborado, como el pretérito pluscuamperfecto. Así, en situaciones en que la alternancia es posible, como en el ejemplo Le dio el regalo que le compró / Le dio el regalo que le había comprado, los estudiantes tienden al uso de las formas simples frente a las compuestas. ¿Cómo propiciar en el aula la reflexión sobre el uso de las distintas formas verbales? Para ello, se diseñó una secuencia didáctica de gramática ( $4^{\circ} \mathrm{ESO}, 15-16$ años) de forma que los estudiantes tomaran conciencia de los distintos tiempos verbales, de sus características y de las diferencias entre ellos, como 
paso previo a su uso consciente en la escritura de un relato sobre una experiencia vivida.

La SDG se organizó en tres fases: en la primera (preparación), se plantea el problema que han de estudiar los alumnos a partir de la pregunta inicial (¿para qué nos sirven los tiempos del pasado?) y se realiza una primera exploración en narraciones de géneros orales (entrevista radiofónica) y escritos (relato literario). En esta observación se apela exclusivamente a la capacidad analítica de los estudiantes como hablantes familiarizados con estos usos. En la segunda fase (realización), se plantea la reflexión explícita sobre los contenidos gramaticales propiamente dichos, las nociones de tiempo y aspecto verbal y las relaciones que las distintas formas verbales establecen en el discurso (anterioridad, simultaneidad, posterioridad; acción acabada o inacabada). El ejemplo utilizado para mostrar el uso de los tiempos verbales del pasado fue el siguiente: Fui al cine el domingo, como hacía siempre; llovía y había quedado con Luis y Alfredo en el parque, donde también nos reuniríamos con Marga y su prima, después de la película. En esta fase los alumnos habían de realizar las dos tareas clave para la investigación: la primera, de conceptualización, en la que habían de explicar a sus compañeros las diferencias entre dos tiempos verbales del pasado; la segunda, de uso reflexivo, en la que habían de escribir una narración sobre un hecho vivido en la que utilizaran el saber gramatical previo sobre los distintos tiempos del pasado. Por último, en la tercera fase, los alumnos habían de sistematizar los aprendizajes de la SDG en un informe que respondiera a la pregunta inicial de la SDG, ¿para qué nos sirven los tiempos verbales del pasado?

Como hemos indicado, el núcleo de la investigación se centró en las tareas realizadas en la segunda fase, porque en ella es donde se planteaba la relación entre el estudio de los tiempos verbales del pasado (conceptualización) y su uso reflexivo en una tarea de escritura.

\section{3 - La noción de subjuntivo}

La segunda investigación tiene como objetivo describir el conocimiento construido por estudiantes de 12 a 16 años sobre el modo verbal en español, entender cómo conceptualizan el subjuntivo y qué valores le otorgan. Para ello, se plantea una actividad de reflexión en torno al contraste entre cuatro pares de oraciones en las que solo cambia el modo verbal. El corpus de oraciones seleccionadas responde en cada caso a una casuística lingüística diferente, tanto en lo que se refiere a las estructuras sintácticas como por el valor que el subjuntivo tiene en cada una de ellas (cuadro 1). 


\begin{tabular}{|l|l|}
\hline Oraciones & Cuestión lingüística planteada \\
\hline $\begin{array}{l}\text { (1a) Busco a un } \\
\text { alumno que sepa } \\
\text { italiano } \\
\text { (1b) Busco a un } \\
\text { alumno que sabe } \\
\text { italiano }\end{array}$ & $\begin{array}{l}\text { Alternancia modal en las oraciones de relativo especificativas. } \\
\text { (la) El hablante busca a alguien que sepa italiano. No está } \\
\text { pensando en una persona concreta sino en una persona } \\
\text { cualquiera que tenga esta característica. } \\
\text { (1b) El hablante busca a una persona concreta a la cual } \\
\text { identifica por los conocimientos que tiene sobre la lengua } \\
\text { italiana, pero no quiere decir que la busque por ese motivo. }\end{array}$ \\
\hline $\begin{array}{l}\text { (2a) Aunque cueste } \\
\text { caro me lo voy a } \\
\text { comprar } \\
\text { (2b) Aunque cuesta } \\
\text { caro me lo voy a } \\
\text { comprar }\end{array}$ & $\begin{array}{l}\text { Alternancia modal en las oraciones subordinadas concesivas. } \\
\text { (2a) El hablante no sabe el valor de aquello que se quiere } \\
\text { comprar o no lo considera relevante. } \\
\text { (2b) El hablante tiene información sobre el precio, sabe que es } \\
\text { caro, pero aún así se lo comprará. }\end{array}$ \\
\hline $\begin{array}{l}\text { (3a) Quisiera una } \\
\text { barra de pan } \\
\text { (3b) Quiero una barra } \\
\text { de pan }\end{array}$ & $\begin{array}{l}\text { Alternancia modal en las oraciones simples. Se pueden } \\
\text { interpretar de manera diferente según el contexto. } \\
\text { (3a) Puede ser la verbalización de un deseo difícil de realizar o } \\
\text { una petición concreta con un marcado valor de cortesía. } \\
\text { (3b) Puede ser la verbalización de un deseo realizable o una } \\
\text { demanda concreta formulada como una aserción. }\end{array}$ \\
\hline $\begin{array}{l}\text { (4a) Quiero que me lo } \\
\text { cuentes } \\
\text { (4b) * Quiero que me } \\
\text { lo cuentas }\end{array}$ & $\begin{array}{l}\text { La alternancia modal no es posible en el caso de oraciones } \\
\text { subordinadas sustantivas introducidas por verbos volitivos } \\
\text { como querer, el cual selecciona sintácticamente el modo } \\
\text { subjuntivo. La oración (4b) es agramatical. }\end{array}$ \\
\hline
\end{tabular}

Cuadro 1 - Oraciones y cuestión lingüística que se plantea (Durán 2017)

Los estudiantes (53 alumnos de tres grupos-clase de $1^{\circ}, 2^{\mathrm{a}}$ y $4^{\mathrm{a}}$ de la ESO) deben discutir en pequeño grupo las diferencias entre cada par mínimo (Bosque 1994) y escribir un pequeño informe con la ayuda de una pauta que guía la reflexión y que les permite orientar la mirada tanto hacia aspectos morfosintácticos como semánticos y pragmáticos. No se trata, como en la investigación anterior, de una SDG completa sino de una actividad exploratoria que tiene como objetivo hacer emerger los conocimientos previos sobre el modo verbal y activar la reflexión metalingüística.

Cabe señalar que la noción modo verbal es muy compleja y abstracta, en primer lugar, porque no se apoya en distinciones léxicas (como sucede con otras categorías como los adverbios, por ejemplo) sino gramaticales, pero también porque el valor concreto que asume depende del contexto en que se utiliza y de la correlación que establece con otros elementos de la oración (Pérez Saldanya 2017: 38). La selección del modo verbal (indicativo, subjuntivo e imperativo en español) se relaciona con la modalidad oracional y con los actos de habla (aseverar, ordenar, rogar, pedir información...). En el caso del subjuntivo, es un modo marcado respecto al indicativo y esas diferencias de marcaje tienen repercusiones 
sintácticas y semánticas. En los contextos sintácticos en los que los dos modos son posibles, las diferencias semánticas son muy sutiles (como bien saben los estudiantes de español como LE).

Los estudiantes, por lo tanto, tienen que tener en cuenta aspectos diversos a la hora de conceptualizar el subjuntivo. Para poder hablar sobre las diferencias entre dos oraciones en las que solo ha cambiado el modo verbal, tienen que poner en marcha conocimientos metalingüísticos de diferente tipo, tanto del ámbito de la palabra y de la oración como del ámbito del discurso. Ahora bien, ¿cómo movilizan estos conocimientos?, ¿cómo utilizan los referentes lingüísticos en la reflexión sobre estas oraciones?, ¿qué uso hacen de la terminología gramatical?, ¿qué relaciones establecen entre el uso lingüístico y el conocimiento sobre el sistema?

\section{4 - La reflexión metalingüística de los estudiantes sobre el verbo}

No es nuestro objetivo detallar en este artículo los resultados de cada una las investigaciones ${ }^{3}$, sino mostrar algunas constantes sobre cómo elaboran los estudiantes de Secundaria Obligatoria las nociones gramaticales sobre el verbo y cómo las utilizan para reflexionar sobre la lengua en actividades de comprensión y de producción.

Hemos organizado estas constantes en torno a cuatro cuestiones: el tipo de actividad metalingüística que realizan los estudiantes en torno al verbo, el papel de los conocimientos sobre los tiempos verbales en la revisión sobre su uso en textos escritos, la relación entre significado y contexto para explicar diferencias de forma entre indicativo y subjuntivo y, por último, las dificultades de los estudiantes para reconocer las nociones gramaticales en el uso de la lengua. Para mostrar estas cuestiones, hemos seleccionado varios ejemplos (dos de cada investigación).

\section{1 - La intensa actividad metalingüística de los estudiantes en la discusión sobre los pares mínimos y las dificultades del metalenguaje}

En ambas investigaciones se observa una intensa actividad metalingüística de los estudiantes mientras realizan las tareas propuestas. Concretamente, en la tarea de reflexión sobre pares mínimos, los resultados muestran que el dispositivo didáctico es útil para poner en marcha la actividad metalingüística de los estudiantes, para activar sus conocimientos previos y para conectar con sus conocimientos gramaticales implícitos; en definitiva, para hacer emerger la reflexión. Muestra de ello es la multiplicidad de criterios que los estudiantes utilizan para justificar la alternancia modal: formales, pero también semánticos y pragmáticos (cuadro 2).

\footnotetext{
${ }^{3}$ Para una visión más detallada de las mismas, v. Rodríguez Gonzalo (2013; 2014 a; 2014b; 2015; 2017), Durán (2012b; 2015a; 2015b; 2017), y Casas, Durán \& Fontich (2017).
} 


\begin{tabular}{|c|c|c|c|c|c|c|}
\hline \multicolumn{7}{|c|}{$\begin{array}{l}\text { (4a) Quiero que me lo cuentes } \\
(4 \mathrm{~b}) * \text { Quiero que me lo cuentas }\end{array}$} \\
\hline \multicolumn{2}{|l|}{ Categoría } & Descripción & $\begin{array}{l}\text { Ejemplos } \\
\text { curso/grupo }\end{array}$ & $\mathrm{n}$ & \multicolumn{2}{|l|}{$\%$} \\
\hline \multicolumn{2}{|c|}{$\begin{array}{l}\text { NORMA } \\
\text { (agramaticalidad) }\end{array}$} & $\begin{array}{l}\text { Los estudiantes } \\
\text { señalan que una de } \\
\text { las formas verbales } \\
\text { no se ajusta a la } \\
\text { norma gramatical. }\end{array}$ & $\begin{array}{l}\text { La diferencia entre } \\
\text { las oraciones } 4 \mathrm{a} y \\
4 \mathrm{~b} \text { consiste en que } \\
\text { la } 4 \mathrm{~b} \text { no es correcta } \\
\text { [...]. (1D) }\end{array}$ & 12 & \multicolumn{2}{|l|}{$31,6 \%$} \\
\hline \multirow{3}{*}{ Formal } & $\begin{array}{l}\text { Fonológica/ } \\
\text { Gráfica }\end{array}$ & $\begin{array}{l}\text { Observan un } \\
\text { cambio formal } \\
\text { circunscrito a } \\
\text { cómo se escribe } \\
\text { la palabra que } \\
\text { cambia. }\end{array}$ & $\begin{array}{l}\text { La diferencia entre } \\
\text { las oraciones } 4 \mathrm{a} \text { y } 4 \mathrm{~b} \\
\text { consiste en que están } \\
\text { escritas diferente } \\
{[\ldots . .](4 \mathrm{~A})}\end{array}$ & 3 & $7,9 \%$ & \multirow{3}{*}{$39,5 \%$} \\
\hline & Morfológica & $\begin{array}{l}\text { Observan } \\
\text { un cambio } \\
\text { morfológico y lo } \\
\text { constatan: hacen } \\
\text { referencia a la } \\
\text { categoría verbo } \\
\text { o al paradigma } \\
\text { verbal. }\end{array}$ & $\begin{array}{l}\text { La diferencia entre } \\
\text { las oraciones } 4 \mathrm{a} \text { y } 4 \mathrm{~b} \\
\text { consiste en cambiar } \\
\text { la conjugación del } \\
\text { verbo (2B) }\end{array}$ & 6 & $15,8 \%$ & \\
\hline & Sintáctica & $\begin{array}{l}\text { Observan un } \\
\text { cambio que tiene } \\
\text { que ver con } \\
\text { las relaciones } \\
\text { sintácticas entre } \\
\text { las palabras de la } \\
\text { oración. }\end{array}$ & $\begin{array}{l}{[\ldots] \text { en la frase la }} \\
\text { palabra "cuentas" } \\
\text { no encaja con las } \\
\text { demás. (1D) }\end{array}$ & 6 & $15,8 \%$ & \\
\hline \multirow{4}{*}{ Semántica } & $\begin{array}{l}\text { modalidad } \\
\text { oracional }\end{array}$ & $\begin{array}{l}\text { Refleja la actitud } \\
\text { del hablante a } \\
\text { través de unas } \\
\text { estructuras } \\
\text { lingüísticas } \\
\text { determinadas. }\end{array}$ & $\begin{array}{l}{[\ldots] \text { En la segunda }} \\
\text { frase el verbo } \\
\text { "cuentas", se podría } \\
\text { poner en una frase } \\
\text { condicional, pero no } \\
\text { en una desiderativa. } \\
(4 \mathrm{~A})\end{array}$ & 2 & $5,3 \%$ & \\
\hline & específico & $\begin{array}{l}\text { El indicativo } \\
\text { selecciona el rasgo } \\
\text { +específico y el } \\
\text { subjuntivo el rasgo } \\
\text {-específico. }\end{array}$ & $\begin{array}{l}\text { Este cambio se } \\
\text { produce para indicar } \\
\text { que la frase } 4 \mathrm{~b} \text { es } \\
\text { algo más concreto. } \\
(4 \mathrm{E})\end{array}$ & 1 & $2,6 \%$ & \multirow{3}{*}{$21,1 \%$} \\
\hline & temporalidad & $\begin{array}{l}\text { Detectan un } \\
\text { cambio de } \\
\text { significado } \\
\text { relacionado con el } \\
\text { tiempo cronológico } \\
\text { extralingüístico al } \\
\text { que hace referencia } \\
\text { el enunciado. }\end{array}$ & $\begin{array}{l}{[\ldots] \text { porque cuentes }} \\
\text { es que lo cuentes } \\
\text { en algún momento } \\
\text { indefinido. Y cuentas } \\
\text { es en el momento. } \\
\text { (2E) }\end{array}$ & 4 & $10,5 \%$ & \\
\hline & homonimia & $\begin{array}{l}\text { Consideran que } \\
\text { el verbo tiene } \\
\text { dos significados } \\
\text { diferentes } \\
\text { (diferencia } \\
\text { semántica del } \\
\text { lexema verbal) }\end{array}$ & $\begin{array}{l}\text { La diferencia entre } \\
\text { las oraciones } 4 \mathrm{a} \text { y } 4 \mathrm{~b} \\
\text { consiste en uno se } \\
\text { refiere a explicar y el } \\
\text { otro a contar. (2A) }\end{array}$ & 1 & $2,6 \%$ & \\
\hline
\end{tabular}




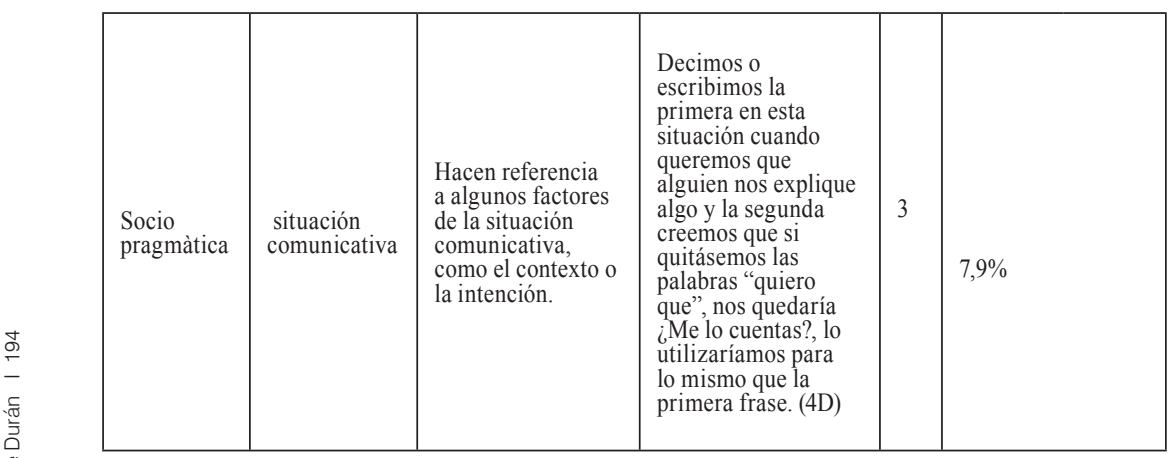

Cuadro 2 - Criterios de caracterización de la alternancia modal indicativo/subjuntivo en 4a y 4b (Durán 2013)

Ahora bien, la actividad metalingüística que se infiere de las respuestas escritas no es homogénea ni entre grupos de estudiantes ni en los textos de un mismo grupo. Los textos que han elaborado los estudiantes muestran grados de conciencia sobre la lengua, de abstracción y de explicitación diferentes, desde comentarios en que justifican la selección modal a partir del conocimiento implícito de la lengua que tienen como usuarios (por ejemplo, los juicios sobre la gramaticalidad o agramaticalidad de las oraciones $4 \mathrm{a}$ y $4 \mathrm{~b}$ en que algunos estudiantes afirman que la frase agramatical *Quiero que me lo cuentas no es correcta porque "no suena nada bien") hasta respuestas que muestran un nivel de conciencia elevado y un alto nivel de abstracción como en esta respuesta de un grupo de estudiantes de $4^{\circ}$ de la ESO (16 años):

\section{Ejemplo 1: Justificación de la diferencia entre 4 a y 4 b (grupo 4A)}

"la segunda [4b] quiere expresar lo mismo pero la frase no está bien construida. En la segunda frase, el verbo 'cuentas' se podría poner en una frase condicional, pero no en una desiderativa"

En sus reflexiones, los estudiantes dejan entrever que poseen un conocimiento declarativo de cómo funciona el sistema lingüístico muy frágil. Utilizan poco el metalenguaje para hablar sobre la lengua y cuando lo hacen no siempre es garantía de que hayan entendido los conceptos que hay detrás de los términos, como se puede observar en este fragmento:

Ejemplo 2: Justificación de la diferencia entre 4 a y 4 b (grupo 4B):

[En (4b) *Quiero que me lo cuentas] no concuerda la primera parte de la oración compuesta con la segunda parte, ni en género ni en número

Hablan de concordancia de género y número para explicar que en *Quiero que me lo cuentas el verbo debería regir subjuntivo, es decir que 
hacen referencia a una exigencia sintáctica pero equivocan el metalenguaje y muestran que no han comprendido del todo los conceptos a los que hacen referencia. En este caso el metalenguaje que no se asienta en una verdadera comprensión de la noción no les es útil para poder reflexionar sobre la lengua. $\mathrm{O}$, dicho de otra manera, el hecho de usar una terminología específica no implica necesariamente una mayor comprensión metalingüística si no se ha asegurado previamente la comprensión de las nociones a las que hacen referencia dichos términos.

Por otro lado, también se percibe un uso del lenguaje común para hablar sobre la lengua:

Ejemplo 3: Justificación de la diferencia entre 4 a y 4 b (grupo 1D):

[...] en la frase [*Quiero que me lo cuentas] la palabra "cuentas" no encaja con las demás

En este caso en el que los estudiantes reflexionan sobre una oración claramente agramatical, se puede observar que los estudiantes recurren a un lenguaje común para explicar los problemas que detectan y que afectan a la sintaxis de la oración, aunque lo hagan de una manera vaga e imprecisa. La sintaxis es percibida como un grupo de palabras que tienen que encajar entre ellas.

Como hemos dicho más arriba, el lenguaje específico es escaso e infructuoso. Los estudiantes muestran dificultades para nombrar los fenómenos que detectan y ello les dificulta la posibilidad de articular el conocimiento claramente. A pesar de ello, muchos de los estudiantes son conscientes de la agramaticalidad de la oración y, como no tienen herramientas terminológicas para justificar dicho fenómeno, el lenguaje común ocupa un lugar importante que suple en cierta manera la falta de términos específicos y permite la reflexión a la vez que muestra las limitaciones.

\section{2 - Los conocimientos gramaticales en la revisión del uso de los tiempos verbales del pasado en una tarea de escritura}

En la investigación sobre el uso reflexivo de los tiempos verbales del pasado, los estudiantes han de revisar el uso que se hace de estos tiempos en la primera versión del texto de sus compañeros (revisión entre iguales), para ver si utilizan formas de uso elaborado, como el pluscuamperfecto. En esta situación, las interacciones de los estudiantes con la profesora muestran el tipo de reflexión metalingüística que desencadena esta actividad, en la que se combinan de forma desigual observaciones de identificación de formas verbales, de evaluación del uso y de modificación.

En el ejemplo 4 observamos la revisión que la estudiante Sara G realiza de la primera versión del relato de su compañero Sebas. Esta revisión se realiza en dos sesiones diferentes. En la primera, la profesora recuerda que 
han de identificar los tiempos verbales utilizados en los relatos, valorar si están bien utilizados y proponer las modificaciones que considerasen. Entre la primera sesión y la segunda, la profesora recuerda con los estudiantes los distintos tiempos verbales del pasado sobre los que han trabajado en la SDG.

Ejemplo 4a. Primera versión del relato de Sebas.

Eraelveranode 2005, tenía 15 añosyvenía de Colombia, todoloquevivíeneseveranofue algo nuevo, porque conocídiferentes sitios, buenoexactamentefuerondos, CastellónyZaragoza.

Digo que este fue el "mejor" verano porque lo pasé junto a mi madre a la que hacía un año que no la veía, no es mucho tiempo pero se la echaba mucho de menos. [...]

En la sesión octava, Sara lee el relato de su compañero y comenta con la profesora:

Ejemplo 4b. Sesión octava SDG. Revisión del relato de Sebas. Interacción de Sara G con la profesora

\begin{tabular}{|c|c|c|}
\hline & SESIÓN OCTAVA & \\
\hline Turno & Alumna & Profesora (PR) \\
\hline 1 & $\begin{array}{l}\text { SARA G: ((refiriéndose al relato que está } \\
\text { revisando)) yo aquí no cambiaría nada } \downarrow\end{array}$ & \\
\hline 2 & & PR: no $\uparrow$ \\
\hline 3 & SG: yo, yo lo veo bien $\downarrow$ & \\
\hline 4 & & PR: lo ves todo bien $\uparrow$ \\
\hline 5 & $\begin{array}{l}\text { SG: yo, sí, porque yo lo habría escrito así también } \\
\downarrow\end{array}$ & \\
\hline 6 & & $\begin{array}{l}\text { PR: [leyendo el relato] } \\
\text { bien, pues si lo ves } \\
\text { bien... }\end{array}$ \\
\hline
\end{tabular}

La estudiante, tras identificar las formas verbales del relato de su compañero, se dirige a la profesora y hace dos comentarios de evaluación ("yo aquí no cambiaría nada", “yo lo veo bien") que justifica en el 
turno 5 ("porque yo lo habría escrito así también"). Esta justificación muestra cómo la alumna revisa acudiendo a criterios de significado, que relaciona con su propio uso. No percibe que solo aparecen dos tiempos (pretérito imperfecto -era, tenía- y pretérito perfecto simple -conocí, fue, pasé-), aquellos más vinculados al uso espontáneo de la lengua.

Ante esta situación, la profesora revisa de nuevo con los estudiantes los distintos tiempos del pasado estudiados en la SDG. Tras ello, Sara continúa revisando el relato:

Ejemplo 4b. Sesión novena SDG. Nueva revisión del relato de Sebas. Interacción de Sara $G$ con la profesora

\begin{tabular}{|l|l|l|}
\hline Turno & SESIÓN NOVENA & \\
\hline 1 & $\begin{array}{l}\text { SARA G: ((dirigiéndose a la profesora, con tono } \\
\text { enfático) POR FAVOR:: QUE NO UTILIZA } \\
\text { NADA ESTE CHICO: ESTÁ TODO EL } \\
\text { RATO::.: }\end{array}$ & Profesora (PR) \\
\hline 2 & $\begin{array}{l}\text { SARA G: A VER: SEBAS - ((con tono burlón } \\
\text { de recriminación, entre risas)) }\end{array}$ & PR: pues escríbeselo $\downarrow$ \\
\hline 3 & $\begin{array}{l}\text { SARA G: ehhh } \uparrow \text { solo utilizas ((como si lo } \\
\text { estuviera escribiendo)) pretérito perfecto simple } \\
\text { no } \uparrow\end{array}$ & $\begin{array}{l}\text { PR: venga: venga: } \\
\text { escríbeselo } \downarrow\end{array}$ \\
\hline 4 & & \\
\hline 5 & &
\end{tabular}

Sara, esta vez, se fija en la forma de los tiempos verbales ("iiipor favor, que no utiliza nada este chico!!!), pero solo advierte a su compañero ("a ver, Sebas, solo utilizas...”), sin proponer modificaciones en el texto. La estudiante advierte realidades formales en las que nunca había reparado lo que revela una mirada diferente sobre el texto, más allá del significado. El lenguaje se ha convertido en "opaco", de ahí la importancia de la actividad metalingüística que la alumna realiza, a pesar de las limitaciones. Sin embargo, esta toma de conciencia formal revierte en su conciencia como escritora y en el uso consciente que hace del pluscuamperfecto en la segunda versión de su propio relato, realizada tras la revisión entre iguales: 
Ejemplo 4c. Primera y segunda versión del relato de Sara G

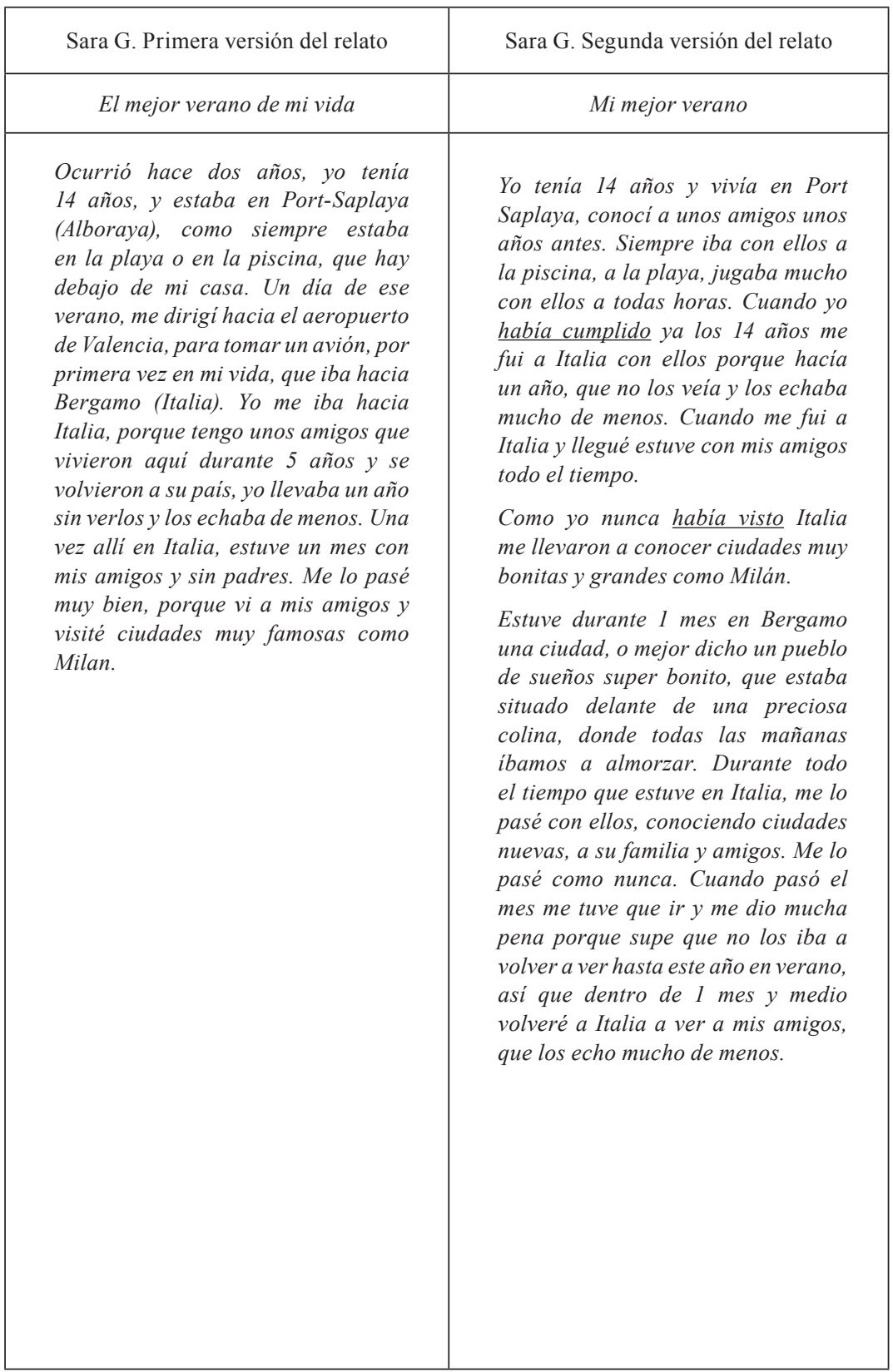


El ejemplo mostrado nos permite observar cómo la revisión favorece la toma de conciencia sobre la forma del texto y permite el uso consciente de recursos gramaticales previamente estudiados. Es decir, la intervención didáctica planteada en la SDG favorece que la actividad metalingüística pase de la manipulación centrada en el significado a la reflexión centrada en la forma. Representamos este proceso en la siguiente figura:

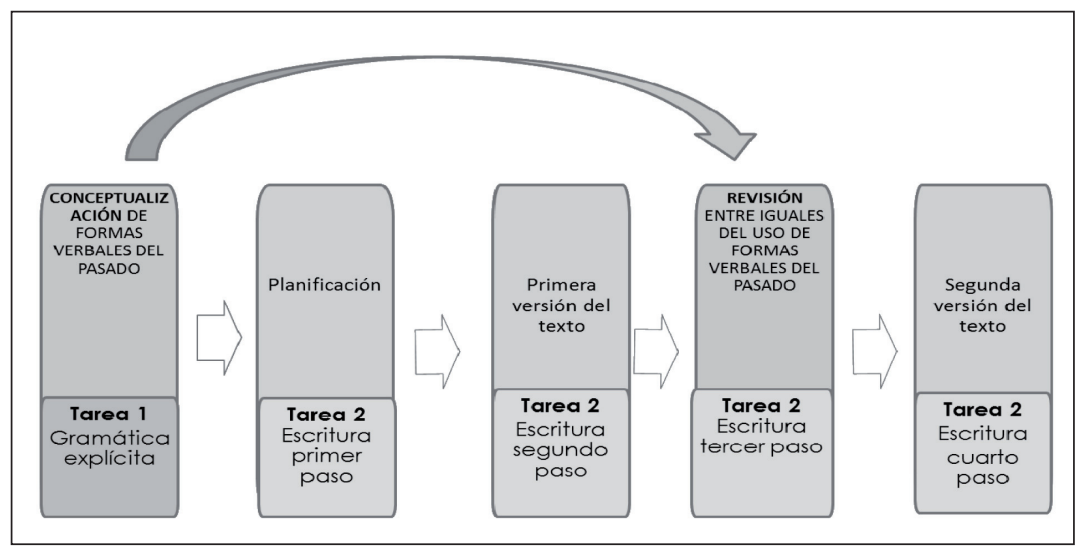

Cuadro 3 - Relación entre conceptualización (tarea 1) y uso reflexivo (tarea 2) en la SDG (Rodríguez Gonzalo 2015, adaptación)

\section{3 - El significado y el contexto para explicar diferencias de forma en los pares mínimos}

Para poder reflexionar sobre las oraciones, lo primero que hacen los estudiantes es llevarlas a un contexto de uso para poder comprenderlas, como se puede ver en el texto de estos estudiantes de $1^{\circ}$ de Secundaria:

Ejemplo 5: Justificación de la diferencia entre los enunciados 3 a y 36 (grupo 1B).

La diferencia entre las oraciones 3a y 3b [Quisiera una barra de pan / Quiero una barra de pan] consiste en que la oración 3a habla de usted y la oración 3b no. [...] significan lo mismo porque solo cambia la manera de decirlo. [...] Decimos o escribimos la primera en esta situación: cuando vamos a comprar el pan y nos encontramos con un señor mayor y la segunda cuando nos encontramos a un joven

En este caso, se trata de oraciones descontextualizadas que, en función del contexto en que aparezcan, pueden tener significados distintos. Para poder reflexionar sobre ellas, remiten a contextos de uso y se imaginan la situación cotidiana de pedir una barra de pan en una panadería. Ello les permite interpretarlas en función de un valor modalizador del subjuntivo (un valor de cortesía) que solo es posible en ese contexto. Pero estas oraciones pueden tener otro significado: la expresión de un deseo difícilmente realizable. 
Es decir, el uso lingüístico aparece como el primer paso para situar el elemento gramatical y es en ese momento cuando los estudiantes son capaces de percibir el modo como una marca de información pragmática que refleja las intenciones del hablante y hacen referencia a su valor de no asertividad o, como en este ejemplo, al valor de cortesía.

Ahora bien, en el camino que va del uso a la reflexión consciente sobre las formas lingüísticas no es inmediato. Estos estudiantes muestran cómo el contexto que se imaginan en que se puede usar una oración determinada hace que olviden la forma que vehicula el contenido y, por lo tanto, anula la posibilidad de observar cuál es el elemento que ha cambiado. Se puede observar esto en la primera parte del fragmento, donde los estudiantes se refieren a un tratamiento de usted que no aparece en el texto, pero que sería posible en el contexto discursivo en que sitúan la oración. El significado pragmático se vuelve tan poderoso que invisibiliza la alternancia modal.

Es decir, observamos que prima el significado y el contexto y que este es un primer paso imprescindible para poder reflexionar sobre la lengua (los estudiantes que no se sitúan en este primer paso no son capaces de iniciar el proceso de reflexión). Ahora bien, cuando el lenguaje se hace transparente, ello obstaculiza la reflexión de los estudiantes sobre la forma que vehicula ese significado. Para observar la forma, por tanto, hay que hacer opaco el lenguaje, en un camino que va del uso a las formas lingüísticas y de estas de nuevo al uso.

\section{4 - Dificultades para reconocer las nociones gramaticales en el uso}

En la investigación sobre el uso reflexivo de los tiempos verbales del pasado, los estudiantes, por parejas, realizan una tarea de conceptualización en la que han de caracterizar y contrastar dos tiempos verbales del pasado. Para ello, siguiendo a Barth (2004), se pedía a los alumnos que identificaran los rasgos o atributos de cada tiempo y que pusieran ejemplos en los que estos rasgos se pusieran de manifiesto. Este contraste se mostraba en un cartel que servía para explicar la tarea a los compañeros.

Los ejemplos que mostramos se refieren a la caracterización del pretérito imperfecto, realizada por dos de las parejas de estudiantes. En el primer caso (ejemplo 6), los alumnos caracterizan el imperfecto con dos rasgos de significado, referidos al tiempo (Para expresar hechos o acciones que ocurren en el pasado) y al aspecto (Presenta la acción en pleno desarrollo sin indicar si terminó). El primero de los ejemplos de uso que incluyen (Mientras escuchaba la charla, me perdía la serie) correlaciona con el primer rasgo, pero el segundo (Tú siempre ibas a pescar con nosotros) alude al valor habitual del pretérito imperfecto, rasgo que ellos no han señalado. Es decir, se observa una cierta desconexión entre los rasgos gramaticales y los ejemplos de uso, lo que muestra dificultades en la conceptualización de este tiempo verbal. 
Ejemplo 6. Caracterización del pretérito imperfecto. Cartel del grupo 2 (detalle)

\begin{tabular}{|c|c|}
\hline & Transcripción \\
\hline $\begin{array}{l}\text { Tiempo verbal 1: } \\
\text {.RBETERIJR. IMPE REEGO............. }\end{array}$ & Tiempo verbal 1: Pretérito imperfecto \\
\hline Aspecto: .IMPERFESTO,.............. & Aspecto: Imperfecto \\
\hline 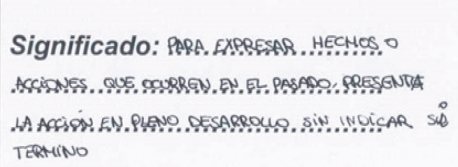 & $\begin{array}{l}\text { Significado: Para expresar hechos o } \\
\text { acciones que ocurren en el pasado. } \\
\text { Presenta la acción en pleno desarrollo } \\
\text { sin indicar si terminó }\end{array}$ \\
\hline Ejemplos de uso (mínimo 2) & Ejemplos de uso (mínimo 2) \\
\hline $\begin{array}{l}\text { 1, Mientras escushaba la sharla. me } \\
\text { perdia ea serie. }\end{array}$ & $\begin{array}{l}\text { 1. Mientras escuchaba la charla, me } \\
\text { perdia la serie. }\end{array}$ \\
\hline 2. Tu. siemere ibas a pescav son nosotros & 2. Tú siempre ibas a pescar con nosotros \\
\hline
\end{tabular}

En el segundo caso (ejemplo 7), los estudiantes también caracterizan el imperfecto aludiendo al tiempo pasado y la acción inacabada (Acciones pasadas en pleno desarrollo y sin término), pero añaden otro rasgo de aspecto, el valor habitual (Hechos o acciones habituales en el pasado). A diferencia del grupo anterior, los estudiantes son conscientes de la relación entre rasgos gramaticales y ejemplos de uso y la señalan mediante los números en superíndice que incluyen en los rasgos de significado. Así, el rasgo de valor habitual se correlaciona con el ejemplo 1.Todos los días se levantaba a las 7 , como se indica con el superíndice que aparece junto al rasgo.

Ejemplo 7. Caracterización del pretérito imperfecto. Cartel del grupo 5 (detalle)

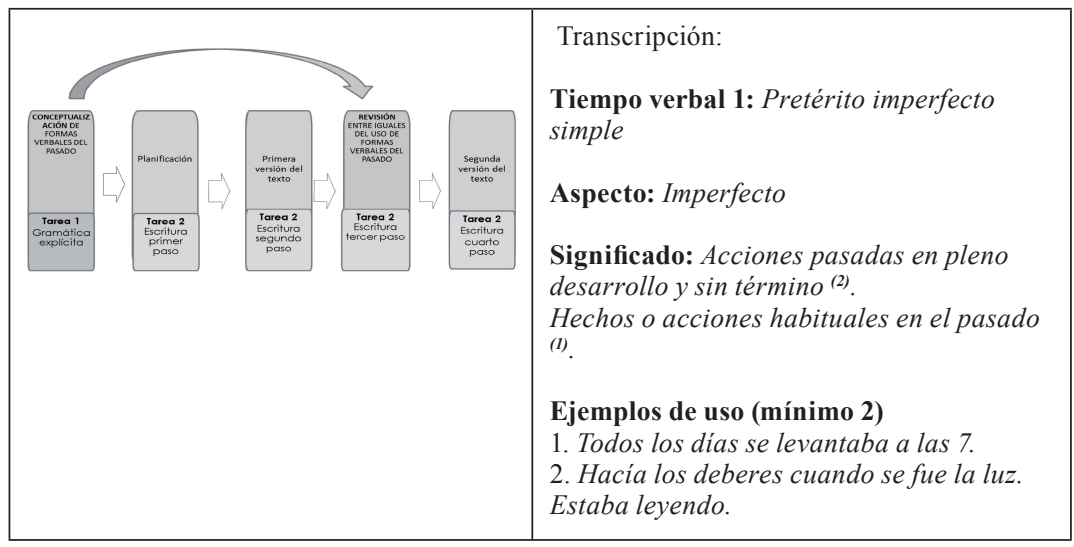


El contraste de los rasgos gramaticales con los ejemplos de uso permite analizar las diferencias de actividad metalingüística en ambos casos. Cuando hay discordancia entre rasgos y ejemplos (ejemplo 7), se ponen de manifiesto las dificultades de los estudiantes para reconocer las nociones gramaticales en el uso. En el ejemplo 7, el imperfecto habitual forma parte del uso de los estudiantes, pero al reflexionar sobre la noción (al conceptualizar), los problemas de relación entre rasgos y ejemplos muestran que no se establece correlación entre definiciones y ejemplos. Esto indica que las definiciones gramaticales carecen de significado real para los alumnos en muchos casos.

\section{5 - Conclusiones e implicaciones didácticas}

Las dos investigaciones que se han presentado de manera sucinta muestran los procesos que siguen los estudiantes en relación con la conceptualización del tiempo y del modo verbal en tareas de reflexión sobre la lengua. Por un lado, la investigación sobre los tiempos verbales del pasado pone de manifiesto el camino de ida y vuelta entre reflexión y uso, observable en las fases de revisión entre iguales y reescritura de los propios textos. Por otro, la investigación sobre el subjuntivo muestra a su vez la capacidad de los estudiantes de reflexionar sobre la lengua desde perspectivas diversas, pero también las dificultades con que se encuentran, dificultades que en líneas generales tienen que ver con la fragilidad con la que han construido sus conocimientos gramaticales sobre el verbo. En ambos casos aparecen denominadores comunes, que nos permiten establecer algunas conclusiones de utilidad en la elaboración de una gramática pedagógica:

a) Los alumnos construyen las nociones a partir de los significados que observan en distintas situaciones de uso. De ahí que los acercamientos formales no sean significativos para ellos. Por tanto, en una gramática pedagógica, las nociones han de abordarse desde situaciones de uso, progresivamente de mayor complejidad, para evitar la fosilización del estereotipo que se observa en buena parte de los libros de texto.

b) El metalenguaje en muchos casos constituye una barrera para acercarse a los conceptos, ya que buena parte de los términos gramaticales son usos específicos de términos de empleo diario (sustantivos como tiempo, modo, aspecto, persona, número o adjetivos como perfecto, imperfecto, entre otros), lo que produce confusiones. En otros casos, son términos integrados en el vocabulario escolar sin una verdadera comprensión de la noción, por lo que resultan poco operativos cuando los estudiantes los necesitan para hablar sobre la lengua. Sin embargo, adquirido el concepto, el término es indispensable (como si fuera el último paso para acotar la noción). Por ello, consideramos que el metalenguaje es necesario para reflexionar sobre cómo funciona el sistema lingüístico. Ahora bien, no se puede empezar por la 
introducción de los términos, como en muchas ocasiones ocurre en las aulas, sino por la comprensión de la noción y ello implica a menudo poder hablar de la lengua con un lenguaje común, ya que, como señala Milian (2005), la enseñanza de la gramática consiste establecer puentes entre el conocimiento intuitivo de la lengua y el conocimiento sistematizado sobre ella y sobre su uso. Si los estudiantes usan un metalenguaje que no comprenden, los términos resultan un obstáculo para la reflexión.

c) Las situaciones de interacción en aula resultan indispensables en una enseñanza de la gramática basada en la reflexión. Ambas investigaciones muestran que la interacción, entre iguales y con el profesor, es muy útil para desencadenar la reflexión sobre la lengua. Como señala Camps (2017: 24), "si no basamos la enseñanza de la gramática en la reflexión, será muy difícil que los alumnos puedan utilizar las definiciones cerradas para comprender cómo funcionan las formas de la lengua en el discurso". Ahora bien, la interacción también tiene limitaciones. Cuando los estudiantes no tienen recursos suficientes (conocimientos previos, ayudas externas...) para resolverlo, el problema se convierte en un callejón sin salida, si no se acompaña de una acción del docente que les guíe en el proceso de sistematización. La comprensión de los conceptos abstractos necesita de un acompañamiento en el proceso de la reflexión a la sistematización.

d) La investigación didáctica muestra la importancia de tener en cuenta las estrategias y las dificultades de los alumnos en el aprendizaje gramatical (en un momento en que las nociones que se están adquiriendo, no están construidas). La enseñanza de la gramática no se puede plantear como un camino unidireccional desde las ciencias del lenguaje a la didáctica de la lengua. Las interacciones entre enseñanza (profesor), aprendizaje (estudiante) y transposición de contenidos muestra un proceso más complejo. De ahí las diferencias entre la gramática pedagógica y las gramáticas divulgativas, que comentábamos al principio.

\section{REFERENCIAS}

Barth, B-M. 2001. L'apprentissage de l'abstraction. París: Retz.

Bosque, I. 1994. Repaso de sintaxis tradicional: ejercicios de autocomprobación. Madrid: Arco Libros.

Bronckart, J.-P. 1985. Las ciencias del lenguaje: ¿un desafío para la enseñanza? Lausana: Unesco.

Camps, A. 2000. Aprendre gramàtica. In: A. Camps; M. Ferrer (coords.). Gramàtica a l'aula. Barcelona: Graó, 101-117.

Camps, A. 2003. Seqüències didàctiques per aprendre gramàtica (SDG). Papers de treball. Departament de Didàctica de la Llengua i la Literatura: Universitat Autònoma de Barcelona, 1. 
Camps, A. 2009. Actividad metalingüística y aprendizaje de la gramática: hacia un modelo de enseñanza basado en la actividad reflexiva. Cultura y Educación. 21(2): 199-214.

Camps, A. 2017. Reflexiones sobre la enseñanza y el aprendizaje de la gramática. In: A. Camp; T. Ribas (coord.). El verbo y su enseñanza. Barcelona: Octaedro, 19-31.

Camps, A.; Zayas, F. (coords.). 2006. Seqüències didàctiques per a aprendre gramàtica. Barcelona: Graó.

Carrasco, A. 1999. El tiempo verbal y la sintaxis oracional. La consecutio temporum. In: I. Bosque; V. Demonte (dirs.). Gramática descriptiva de la lengua española (vol.II). Madrid: Espasa, 3061-3128.

Casas, M. 2014. Interacción, uso lingüístico y construcción del saber gramatical en la educación primaria. Tejuelo, 65-83. Monográfico 10.

Casas, M.; Durán, C.; Fontich, X. 2017. La construcció del coneixement gramatical en aprenents d'educació primària i secundària: algunes aproximacions a l'aprenentatge del verb. [The construction of grammatical knowledge by students of primary and secondary education: some approaches to the learning of the verb]. Caplletra. 63: 111-138.

Chartrand, S.G. 2003. Sept chantiers pour travailler la grammaire en classe. Québec français. 129: 73-76.

Chevallard. 1985. La transposition didactique: du savoir savant au savoir enseigné. Grenoble: La Pensée Sauvage.

Cuenca, M.J.; Hilferty, J. 1999. Introducción a la lingüística cognitiva. Barcelona: Ariel.

Durán, C. 2010. Parlem de l'adverbi: els conceptes gramaticals dels alumnes de l'ESO. Articles de Didàctica de la Llengua i la Literatura. 52: 91-111.

Durán, C. 2012a. El concepto de verbo de los alumnos de secundaria. Representación gramatical y actividad metalingüística. In: Aula de Lingua: Interação e reflexão. Instituto Politécnico de Leira / Universidade do Minho / Universidade de Aveiro: Leiria / Braga / Aveiro, 111-131. Recuperado de http:// repositorium.sdum.uminho.pt/handle/1822/18280.

Durán, C. 2012b. El tractament del mode en els llibres de text. Bellaterra: Journal of Teaching \& Learning Language \& Literature. 5(2): 25-42.

Durán, C. 2013. Els valors del subjuntiu. Un estudi de cas sobre l'activitat metalingüistica dels estudiants de secundària a partir del contrast modal. Tesis doctoral no publicada. Barcelona: Universitat Autònoma de Barcelona. Recuperada de http://hdl.handle.net/10803/129184.

Durán, C. 2015a. The notion of verb mood in students in compulsory secondary education. In: T. Ribas; X. Fontich; O. Guasch (eds.). Grammar at school. Research of Metalinguistic Activity in Language Education. Brussels: Peter Lang, 173-202. 
Durán, C. 2015b. La noción de modo verbal en alumnos de la secundaria obligatoria: concepciones y obstáculos. Tejuelo. 22: 28-49.

Durán, C. 2017. Los conceptos de los estudiantes de secundaria sobre el subjuntivo. In: A. Camps; T. Ribas (coord.). El verbo y cómo enseñarlo. Barcelona: Octaedro, 80-91.

Fisher, C. 2004. La place des représentations des apprenants en didactique de la grammaire. In: D. C. Vargas (dir.). Langue et études de la langue. Approches linguistiques et didactiques. Aix-en-Provence: Publications de l’Université de Provence, 383-393.

Fontich, X. 2006. Hablar y escribir para aprender gramática. Barcelona: ICE-Horsori.

Fontich, X. 2017. Enseñar gramática: cuando el para qué articula el qué y el cómo. Textos de Didáctica de la Lengua y la Literatura. 75: 7-12.

Fontich, X.; Camps, A. 2014. Towards a rationale for research into grammar teaching in schools. Research Papers in Education. Vol.29, 5: 598-625.

Garitte, C. 2004. Les conditions du verbe: aspects cognitifs et développementaux. In: C. Vaguer; B. Lavieu (eds.). Le verbe dans tous ses états. Grammaire, sémantique, didactique. Namur: Presses Universitaires de Namur, 19-32.

Grijelmo, A. 2006. Gramática descomplicada del español. Madrid: Taurus.

van Hiele, P. 1986. Structure and Insight: A theory of mathematics education. Orlando: Academic Press.

Lakoff, G. 1987. Women, fire, and dangerous things. Chicago/London: The University of Chicago Press.

Langacker, R. W. 1987. Foundations of Cognitive Grammar. Vol I: Theoretical Prerequisites. Stanford, Cal.: Stanford University Press.

Lemke, J. L. 1997. Aprender a hablar ciencia. Barcelona: Paidós.

Locke, T. 2010. Beyond the grammar wars. New York: Routledge.

López García, A. 2005. Gramática cognitiva para profesores de español L2. Madrid: Arco Libros.

Lusetti, M. 2008. Le verbe pour commencer la grammaire au CE1. Recherches. 48: 105-135.

Martínez, A. 2010. El tratamiento de la estructura del predicado en una muestra de libros de texto. In: T. Ribas (coord). Libros de texto y enseñanza de la gramàtica. Barcelona: Graó, 135-154.

Milian, M. 2005. Parlar per "fer gramàtica". Articles de Didàctica de la Llengua i la Literatura. 37: 11-30.

Notario, G. 2001. Los conceptos gramaticales de los alumnos de secundaria: el sujeto. In: A. Camps (coord.). El aula como espacio de investigación y reflexión. Barcelona: Graó, 181-193. 
Pérez Saldanya, M. 2017. La lingüística del verbo: el objeto de enseñanza desde las perspectivas morfosintáctica, semántica y pragmática. In: A. Camps \& T. Ribas (coords.). El verbo y su enseñanza. Barcelona: Octaedro, 32-50.

Real Academia Española; Asociación de Academias de la Lengua Española 2011. Nueva gramática básica de la lengua española. Madrid: Espasa.

Rodríguez Gonzalo, C. 2013. L'apprentissage des temps verbaux en espagnol. Relations entre connaissances déclaratives et usage dans les textes. Lidil. 47: 41-59.

Rodríguez Gonzalo, C. 2014a. Reflexive knowledge of the past tenses in Spanish. In: T. Ribas; X. Fontich; O. Guasch (eds.). Grammar at school. Research of Metalinguistic Activity in Language Education. Brussels: Peter Lang, 227-234.

Rodríguez Gonzalo, C. 2014b. Enseñanza de gramática y escritura: el papel de la revisión. Lenguaje y Textos. 40: 19-31.

Rodríguez-Gonzalo, C. 2015. A classroom intervention on Spanish grammar and writing: the use of the past tense with secondary school students. Cultura y Educación. 27(4): 879-898.

Rodríguez Gonzalo, C. 2017. ¿Para qué sirven los tiempos verbales del pasado? El conocimiento gramatical y el dominio expresivo en alumnos de Secundaria. In: A. Camps; T. Ribas (coord.). El verbo y su enseñanza. Barcelona: Octaedro, 92-107.

Roubaud, M. N.; Touchard, Y. 2004. Vers la notion de verbe: De l'approche intuitive à la construction du savoir. In: C. Vargas (dir.). Langue et études de la langue. Approches linguistiques et didactiques. Aix-en-Provence: Publications de l'Université de Provence, 257-267.

Tisset, C. 2004. Un jour fut le verbe. In: C. Vaguer; B. Lavieu (eds.). Le verbe dans tous ses états. Grammaire, sémantique, didactique. Namur: Presses Universitaires de Namur, 33-50.

Torralba, M. 2012. La comprensió dels temps verbals a primària. Estudi descriptiu dels coneixements dels alumnes de quart $i$ sisè sobre el verb com a codificador temporal. Tesis doctoral no publicada. Barcelona: Universitat Autònoma de Barcelona. Recuperada de http://www.tdx.cat/ handle/10803/117021.

Vigotski, L.S. 1995. Pensamiento y lenguaje. Barcelona: Paidós.

Weinrich, H. 1974. Estructura y función de los tiempos del lenguaje. Madrid: Gredos.

Zayas, F. 2011. El lugar de la gramática en la enseñanza de la lengua. In: U. Ruiz (coord.). Lengua Castellana y Literatura. Investigación, innovación y buenas prácticas. Barcelona: Graó, 91-106. 